\title{
Comparison of endoscopic ultrasonography with and without contrast enhancement for characterization of pancreatic tumors: a meta-analysis
}

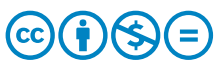

\author{
Authors \\ Institutions \\ 1 Second Department of Internal Medicine, Wakayama \\ Medical University, Wakayama, Japan \\ 2 Clinical Study Support Center, Wakayama Medical \\ University Hospital, Wakayama, Japan \\ 3 Department of Gastroenterology, Jean Mermoz Private \\ Hospital, Ramsay Générale de Santé, Lyon, France \\ 4 University of Bologna/Hospital of Imola, Imola, Italy \\ 5 Department of Internal Medicine (DAIM), Hirslanden \\ Kliniken Beau Site, Salem und Permanence Bern, \\ Switzerland \\ 6 Department of Gastroenterology and Hepatology, \\ Kindai University Faculty of Medicine, Osaka-Sayama, \\ Japan
}

Yasunobu Yamashita', Toshio Shimokawa², Reiko Ashida', Bertrand Napoléon ${ }^{3}$, Andrea Lisotti ${ }^{4}$, Pietro Fusaroli ${ }^{4}$, Rodica Gincul ${ }^{3}$, Christoph F. Dietrich ${ }^{5}$, Shunsuke Omoto ${ }^{6}$, Masayuki Kitano ${ }^{1}$

submitted 8.5.2021

accepted after revision 27.9.2021

published online 25.2.2022

Bibliography

Endosc Int Open 2022; 10: E369-E377

DOI 10.1055/a-1782-5033

ISSN 2364-3722

(c) 2022. The Author(s).

This is an open access article published by Thieme under the terms of the Creative Commons Attribution-NonDerivative-NonCommercial License, permitting copying and reproduction so long as the original work is given appropriate credit. Contents may not be used for commercial purposes, or adapted, remixed, transformed or built upon. (https://creativecommons.org/licenses/by-nc-nd/4.0/)

Georg Thieme Verlag KG, Rüdigerstraße 14,

70469 Stuttgart, Germany

Corresponding author

Masayuki Kitano, MD, PhD, Second Department of Internal Medicine, Wakayama Medical University, 811-1, Kimiidera, Wakayama City, Wakayama 641-0012, Japan

Fax: +81-73-4453616

kitano@wakayama-med.ac.jp $\circledast$ Supplementary material is available under

https://doi.org/10.1055/a-1782-5033

\section{ABSTRACT}

Background and study aims Endoscopic ultrasonography (EUS) is a reliable and efficient modality for detecting pancreatic tumors; however, plain EUS (P-EUS) is limited with respect to characterization of pancreatic tumors. Recently, the use of contrast-enhanced harmonic EUS $(\mathrm{CH}$ EUS) has increased, and its utility for characterization of pancreatic tumors has been reported. This meta-analysis compares the diagnostic ability of P-EUS with that of $\mathrm{CH}$ EUS for characterization of pancreatic tumors.

Methods A systematic meta-analysis of all potentially relevant articles in PubMed, the Cochrane library, and Google Scholar databases was performed. Fixed effects or random effects models were used to investigate pooled sensitivity, specificity, positive likelihood ratio, and negative likelihood ratio, with $95 \%$ confidence intervals (Cls).

Results This meta-analysis included 719 patients who underwent $\mathrm{CH}$-EUS and 723 who underwent P-EUS, from six eligible studies. The pooled estimates of sensitivity, specificity, and diagnostic odds ratio were $93 \%(95 \% \mathrm{Cl}, 0.90-$ 0.95), $80 \%$ (95\% Cl, 0.75-0.85), and 57.9 (95\% Cl, 25.9$130)$, respectively, for $\mathrm{CH}$-EUS, and $86 \%(95 \% \mathrm{Cl}, 0.82-$ $0.89)$, $59 \%(95 \% \mathrm{Cl}, 0.52-0.65)$, and 8.3 (95\% Cl, 2.824.5) for P-EUS. The areas under the summary receiver operating characteristics curves for $\mathrm{CH}$-EUS and P-EUS were 0.96 and 0.80 , respectively. The diagnostic odds ratio for pancreatic cancer was 2.98 times higher on $\mathrm{CH}$-EUS than on P-EUS $(P=0.03)$. Funnel plots demonstrated no publication bias.

Conclusions This meta-analysis demonstrates that $\mathrm{CH}$ EUS has higher diagnostic accuracy for pancreatic cancer than P-EUS, and is thus a valuable tool for characterization of pancreatic tumors. 


\section{Introduction}

Despite the rapid development of imaging modalities, the incidence and mortality rates of pancreatic cancer are rising rapidly, and the disease is now the fourth leading cause of cancerrelated death in the United States and Japan [1,2]. It is usually diagnosed at an advanced stage, and $80 \%$ to $90 \%$ of patients with pancreatic cancer have unresectable tumors. For patients with metastatic disease, the 5 -year survival rate is $<10 \%$ [3]. Therefore, early detection of pancreatic cancer is necessary to improve the prognosis.

In this respect, endoscopic ultrasonography (EUS) is one of the most accurate diagnostic modalities for pancreatobiliary diseases because it offers spatial resolution superior to any other imaging modality [4, 5]. In fact, EUS is significantly superior to computed tomography (CT) for detection and characterization of small pancreatic tumors $[5,6]$ in clinical practice. EUS offers advantages over other imaging modalities for detection and analysis of pancreatic cancer. In a meta-analysis, the pooled estimates of EUS for diagnosing pancreatic malignancy in patients evaluated because of indeterminate contrast-enhanced multi-detector-row CT (MDCT) were a sensitivity of $85 \%$, a specificity of $58 \%$, an accuracy of $75 \%$, and summary receiver operating characteristic (SROC) curve of 0.80 [6].

However, despite its ability to detect small pancreatic tumors with high sensitivity, plain EUS (P-EUS) alone is limited in its ability to distinguish pancreatic cancer from non-neoplastic pancreatic tumors because most pancreatic tumors are detected as hypoechoic tumors [7]. Moreover, approximately $60 \%$ of small pancreatic tumors of $\leq 15 \mathrm{~mm}$ in asymptomatic patients are not pancreatic cancer [8]. Therefore, contrast-enhanced EUS (CE-EUS), which allows evaluation of the tumor vascularity, can help improve their characterization. Previously, endosonographers used contrast-enhanced Doppler EUS, which has limitations due to Doppler-related artifacts such as blooming and overpainting.

Development of contrast harmonic imaging allowed realtime depiction of microvessels and parenchymal perfusion without Doppler-related artifacts. Contrast-enhanced harmonic EUS (CH-EUS) with second-generation contrast agents is now considered an accurate technique for investigation of pancreatic tumors [8]. In fact, previous reports found that the diagnostic ability of $\mathrm{CH}$-EUS was significantly higher than that of contrast-enhanced MDCT and magnetic resonance imaging (MRI) for the characterization of small pancreatic tumors $(\leq 20 \mathrm{~mm})$ in clinical practice $[9,10]$.

Prior to development of $\mathrm{CH}$-EUS, pancreatic tumors were characterized by P-EUS using criteria such as hypoechogenicity and an irregular periphery. However, characterization with PEUS alone was not adequate. Therefore, we evaluated improvements in characterization of pancreatic tumors by adding $\mathrm{CH}$ EUS. In this meta-analysis, we focused on the role of P-EUS in tumor characterization rather than detection. Although four meta-analyses have investigated the diagnostic accuracy of CE-EUS or $\mathrm{CH}$-EUS for characterization of pancreatic tumors [11-14], no meta-analysis has directly compared $\mathrm{CH}$-EUS and P-EUS. Therefore, we performed this meta-analysis to compare these two modalities for the characterization of pancreatic tumors.

\section{Methods}

This study does not involve active human participants and/or animal. Therefore, formal consent, informed consent, institutional review board approval, and ethical approval are not applicable. The review and meta-analysis were developed and reported in accordance with the preferred reporting items for systematic reviews and meta-analyses (PRISMA).

\section{Research methods}

PubMed, the Cochrane library, and Google Scholar databases between January 2000 and February 2021 were searched for relevant English-language articles using the following keywords: ("pancreatic cancer" OR "pancreatic mass" OR "pancreatic carcinoma" OR "pancreatic tumors") AND ("contrast-enhanced" OR "echo enhanced" OR "contrast enhancement") AND ("EUS" OR "endoscopic ultrasonography” OR "endoscopic ultrasound" OR "endosonography”). An expert methodologist (T.S.) oversaw systematic review and meta-analysis. The references of pertinent articles were independently checked to identify any further relevant articles by two authors (Y.Y. and R.A.).

\section{Inclusion and exclusion criteria}

The following were used as inclusion criteria: pancreatic solid masses explored by P-EUS and CH-EUS; the use of second-generation contrast agents; final diagnoses determined by histopathological examination of surgically obtained specimens, EUSTA (tissue acquisition), or clinical follow-up of at least 6 months for benign lesions; provision of suitable data to allow true positive, false positive, false negative, and true negative rates to be obtained; and pancreatic cancer defined by $\mathrm{CH}$-EUS and P-EUS as follows: On CH-EUS, tumors with a hypo-enhanced pattern in which the echo intensity of the tumor was lower than that of the surrounding pancreatic tissue; on P-EUS, tumors showing hypoechogenicity and/or an irregular periphery. All reports using the above definitions were included.

The following exclusion criteria were applied: unavailability of complete data and papers of the following types: conference abstracts, case reports, reviews, and editorials.

\section{Statistical methods}

The pooled estimates of sensitivity, specificity, and diagnostic odds ratios were obtained using Meta-Disc version 1.4. Pooled results were analyzed using a fixed effects model (MantelHaenszel method) when heterogeneity was not present [15], and a random effects model (DerSimonian-Laird method) when heterogeneity was detected [16]. The heterogeneity of the studies was evaluated using the Cochrane $Q$ test and $I^{2}$ statistic. Publication bias was assessed using the funnel plot. Differences were considered statistically significant at $P<0.05$. A SROC was constructed, and the area under the SROC (AUC) was calculated. The Quality Assessment of Diagnostic Accuracy 


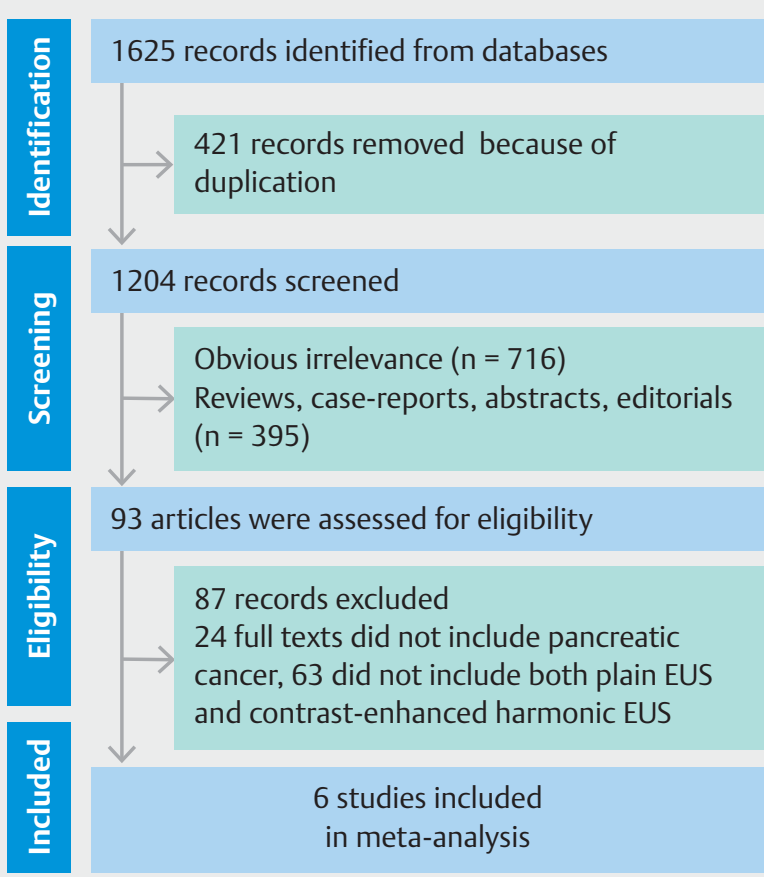

- Fig. 1 Flowchart showing the study selection process.

Studies-2 (QUADAS-2) tool was used to assess the quality of the selected studies by two authors (Y.Y. and R.A.).

\section{Results}

\section{Study selection and quality assessment}

A total of 1625 articles were originally identified in PubMed, the Cochrane library, and Google Scholar databases. After application of the inclusion and exclusion criteria, six articles [17-22] with a total of 719 patients examined with CH-EUS and 723 patients examined with P-EUS were selected for the final analysis ( Fig.1). A flowchart detailing the study selection process is shown in $>$ Fig. 1, and the main characteristics of the studies are listed in $>$ Table 1 . In all adopted reports, characterization was based on pathological evaluations. Moreover, in patients with negative findings after pathological evaluation, the fol- low-up period was set so that benign tumors could be monitored for potential malignancy. QUADAS-2 assessment of the included studies showed that most studies had a low risk of bias ( Fig. 2, > Table 2).

\section{Diagnostic value}

The pooled estimates of sensitivity and specificity for the characterization of pancreatic tumors were $93 \%(95 \% \mathrm{Cl}, 90-95)$ and $80 \%(95 \% \mathrm{Cl}, 75-85)$, respectively, on $\mathrm{CH}$-EUS, and $86 \%$ (95\% Cl, 82-89) and 59\% (95\% Cl, 52-65) on P-EUS (> Fig.3, - Fig. 4). The pooled positive likelihood ratio and negative likelihood ratio were $4.7(95 \% \mathrm{Cl}, 2.7-8.2)$ and $0.11(95 \% \mathrm{Cl}, 0.06-$ 0.16), respectively, on $\mathrm{CH}$-EUS, and 2.16 (95\% Cl, 1.2-3.87) and $0.28(95 \% \mathrm{Cl}, 0.12-0.67)$ on P-EUS ( $>$ Fig. 5, > Fig. 6). The area under the SROC curve was 0.96 on CH-EUS and 0.80 , respectively, on P-EUS ( Fig. 7). $\mathrm{CH}$-EUS and P-EUS were therefore demonstrated as being useful for the characterization of pancreatic tumors, with high pooled diagnostic odds ratios of 57.87 and 8.26, respectively, ( $>$ Fig. 8). However, the pooled diagnostic odds ratio of $\mathrm{CH}$-EUS for diagnostic accuracy of pancreatic cancer was 2.98 times higher than that for P-EUS ( $P=$ 0.03 ; Fig.9). There were statistically significant heterogeneities in the sensitivity of P-EUS, the specificities of $\mathrm{CH}$-EUS and $\mathrm{P}$-EUS, positive likelihood ratios on $\mathrm{CH}$-EUS and $\mathrm{P}$-EUS, negative likelihood ratio on P-EUS, diagnostic odds ratios on $\mathrm{CH}$-EUS and P-EUS, and accuracies of CH-EUS and P-EUS. These pooled results were analyzed using a random effects model. Assessment of publication bias by funnel plots showed no indication of its presence ( $\triangleright$ Fig. 10$)$. Summary data from the meta-analysis are presented in $>$ Table 3.

\section{Discussion}

We attempted to compare corresponding imaging techniques to assess the utility of $\mathrm{CH}$-EUS. Specifically, we tried to compare P-EUS with CT/MR for detection of focal pancreatic masses, and to compare $\mathrm{CH}$-EUS with contrast-enhanced CT/MR. However, there are no reports comparing P-EUS with plain CT or MRI for detection of pancreatic tumors. Also, there were no reports comparing $\mathrm{CH}$-EUS with contrast-enhanced MRI. Therefore, we performed a sub-meta-analysis of $\mathrm{CH}$-EUS and contrast-enhanced CT (three articles, including a total of 542 patients examined with $\mathrm{CH}$-EUS and 458 patients examined with CE-CT,

- Table 1 Characteristics of the selected studies.

\begin{tabular}{|c|c|c|c|c|c|c|c|}
\hline Study & Country & $\begin{array}{l}\text { No.pa- } \\
\text { tients }\end{array}$ & $\begin{array}{l}\text { Sex } \\
(M / F)\end{array}$ & Age & $\begin{array}{l}\text { Contrast } \\
\text { agent }\end{array}$ & $\begin{array}{l}\text { Contrast } \\
\text { mode }\end{array}$ & Gold standard \\
\hline Omoto et al. 2021 & Japan & 204 & $108 / 96$ & 67.9 & Sonazoid & Harmonic & Histology, follow-up (>12 mo) \\
\hline Bunganič et al. 2018 & Czech Republic & 116 & $62 / 54$ & 67.5 & SonoVue & Harmonic & Histology, follow-up (>12 mo) \\
\hline Harmsen et al. 2018 & Germany & 215 & $80 / 135$ & 62 & SonoVue & Harmonic & Histology, follow-up (>12 mo) \\
\hline Uekitani et al. 2016 & Japan & 49 & $23 / 26$ & 66.5 & Sonazoid & Harmonic & Histology \\
\hline Hocke et al. 2012 & Germany & 58 & $39 / 19$ & 60 & SonoVue & Harmonic & Histology, follow-up (12 mo) \\
\hline Fusaroli et al. 2010 & Italy & 90 & $44 / 46$ & 67 & SonoVue & Harmonic & Histology, follow-up (>6 mo) \\
\hline
\end{tabular}




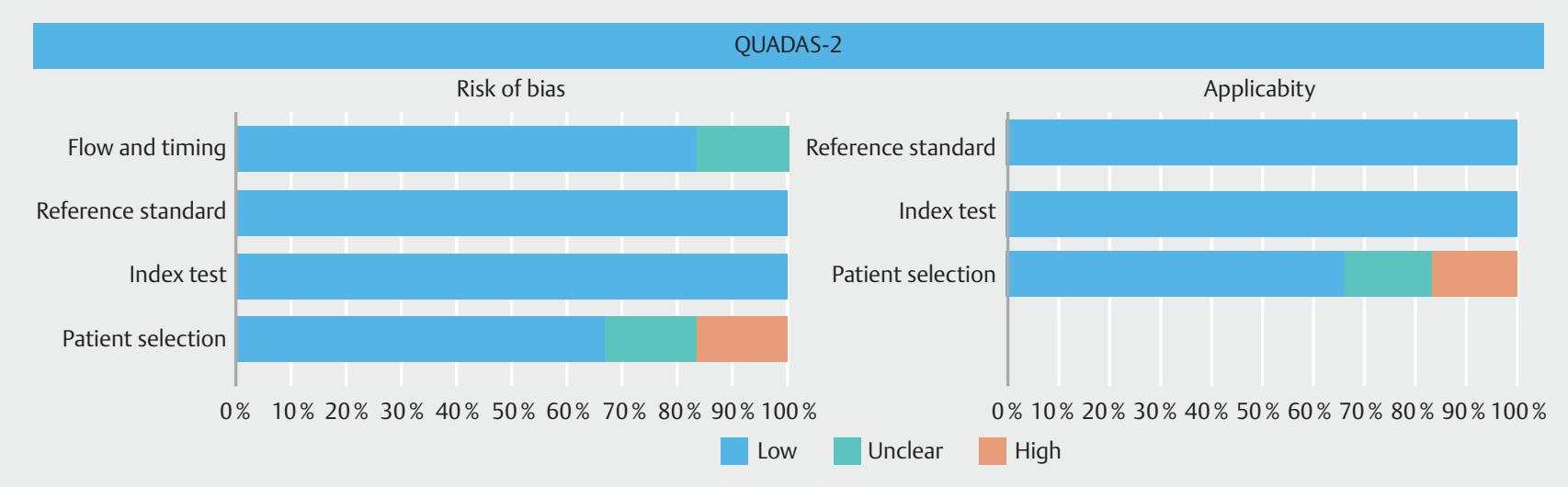

Fig.2 Quality assessment of the studies according to QUADAS-2. Red, high risk of bias; green: unclear risk of bias; blue, low risk of bias.

Table 2 Quality assessment of the included studies according to QUADAS-2.

\begin{tabular}{|l|l|l|l|l|l|l|l|}
\hline & \multicolumn{2}{|l|}{ Risk of bias } & \multicolumn{3}{c|}{ Applicability concerns } \\
\hline Study & $\begin{array}{l}\text { Patient } \\
\text { selection }\end{array}$ & Index test & $\begin{array}{l}\text { Reference } \\
\text { standard }\end{array}$ & $\begin{array}{l}\text { Flow and } \\
\text { timing }\end{array}$ & $\begin{array}{l}\text { Patient } \\
\text { selection }\end{array}$ & $\begin{array}{c}\text { Reference } \\
\text { Indandard }\end{array}$ \\
\hline Omoto et al. 2021 & Low & Low & Low & Low & Low & Low \\
\hline Bunganič et al. 2018 & Low & Low & Low & Low & Low & Low \\
\hline Harmsen et al. 2018 & Low & Low & Low & Unclear & Low & Low \\
\hline Uekitani et al. 2016 & Unclear & Low & Low & Low & Unclear & Low \\
\hline Hocke et al. 2012 & High & Low & Low & Low & High & Low \\
\hline Fusaroli et al. 2010 & Low & Low & Low & Low & Low & Low \\
\hline
\end{tabular}
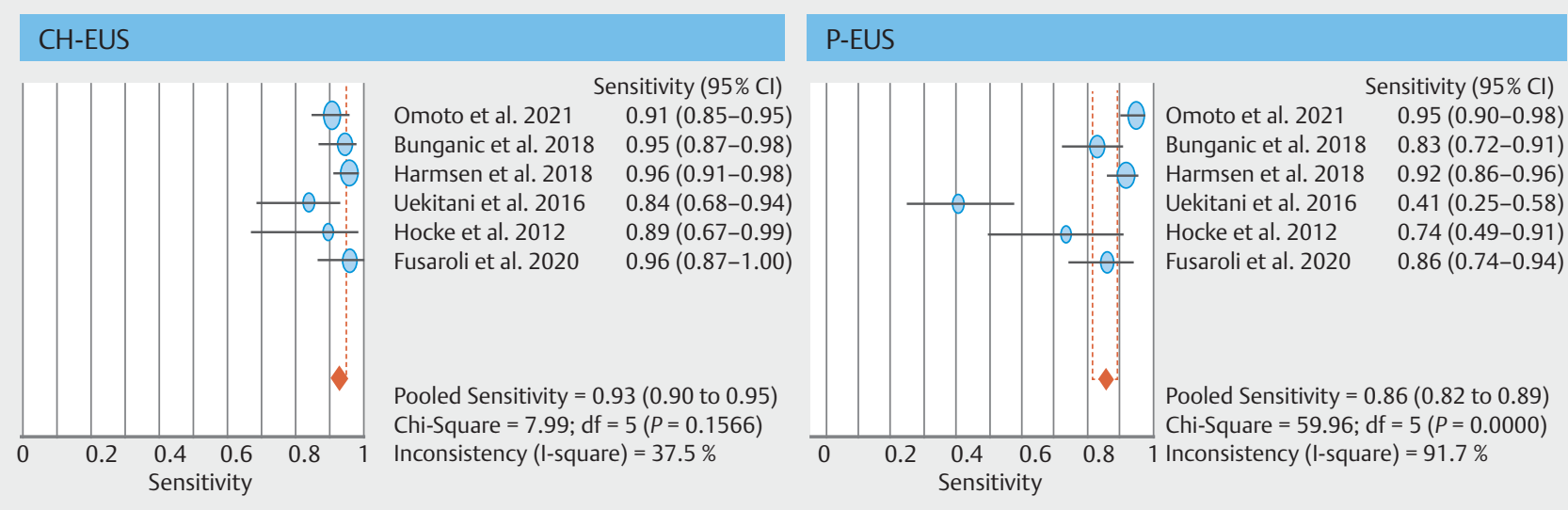

- Fig. 3 Forest plots of pooled sensitivity for characterization of pancreatic tumors with contrast-enhanced harmonic endoscopic ultrasonography (CH-EUS) and plain EUS (P-EUS).

were assessed) (Supplementary file). However, three articles are too small for meta-analysis and no useful results were obtained. Thus, it is necessary to perform meta-analysis with more reports in the future. Therefore, we compared the ability of CH-EUS and P-EUS to characterize pancreatic tumors; to the best of our knowledge, this is the first meta-analysis of this type. All diagnostic parameters assessed in this meta-analysis showed that $\mathrm{CH}$-EUS was superior to P-EUS for characterization of pancreatic tumors.

Four meta-analyses have already investigated the ability of CE-EUS, including contrast-enhanced Doppler EUS and $\mathrm{CH}$ EUS, to diagnose pancreatic cancer. These studies found pooled 


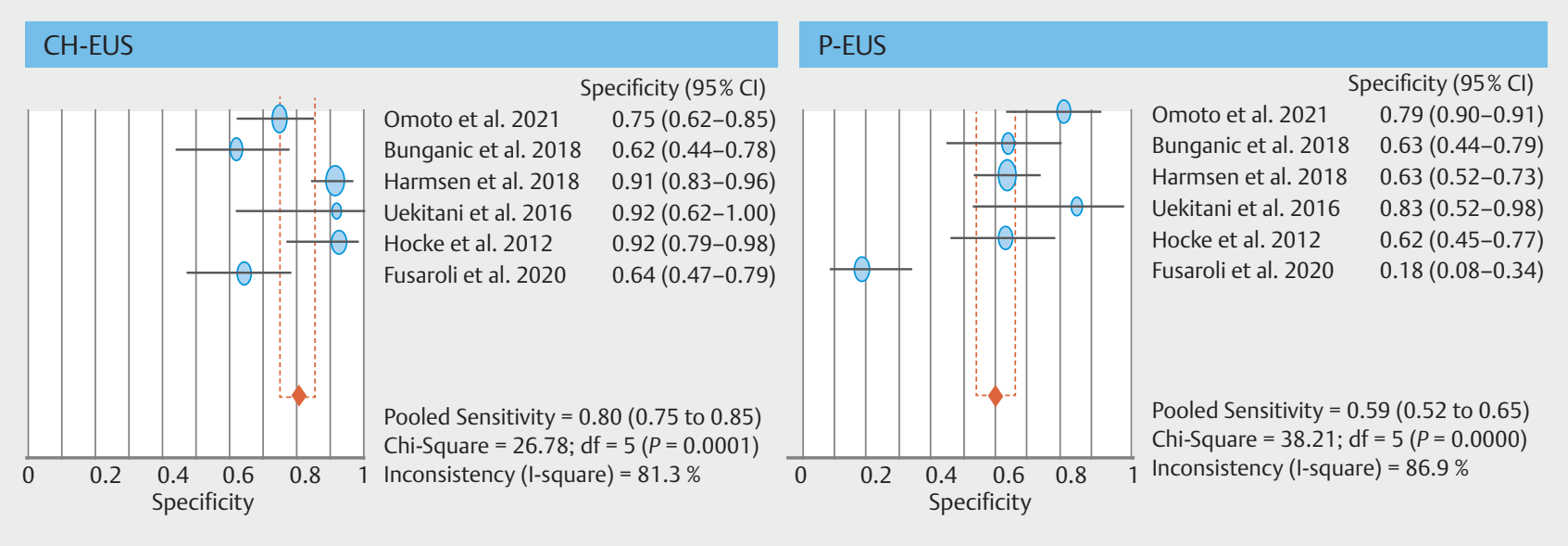

Fig. 4 Forest plots of pooled specificity for characterization of pancreatic tumors on contrast-enhanced harmonic endoscopic ultrasonography (CH-EUS) and plain EUS (P-EUS).

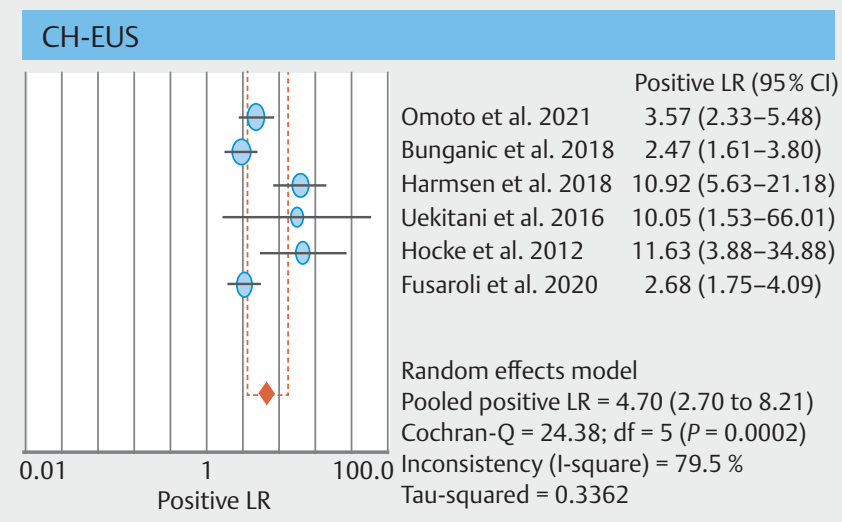

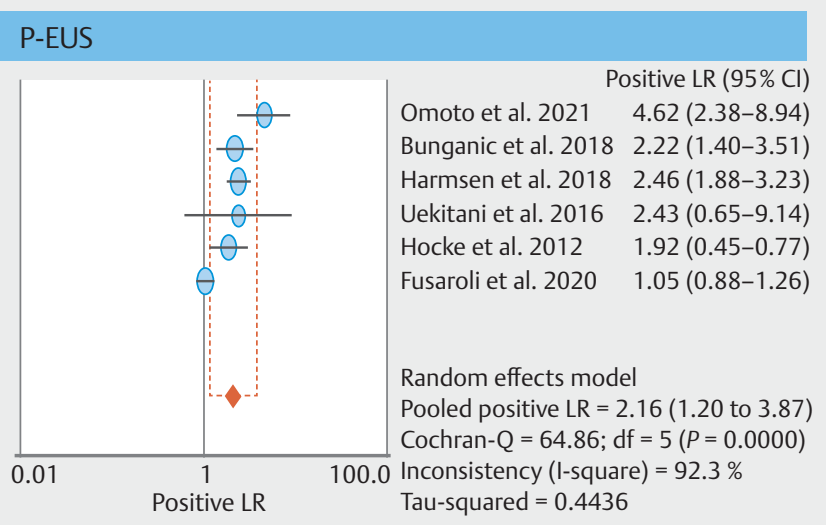

Fig. 5 Forest plots of the pooled positive likelihood ratios on contrast-enhanced harmonic endoscopic ultrasonography (CH-EUS) and plain EUS (P-EUS).
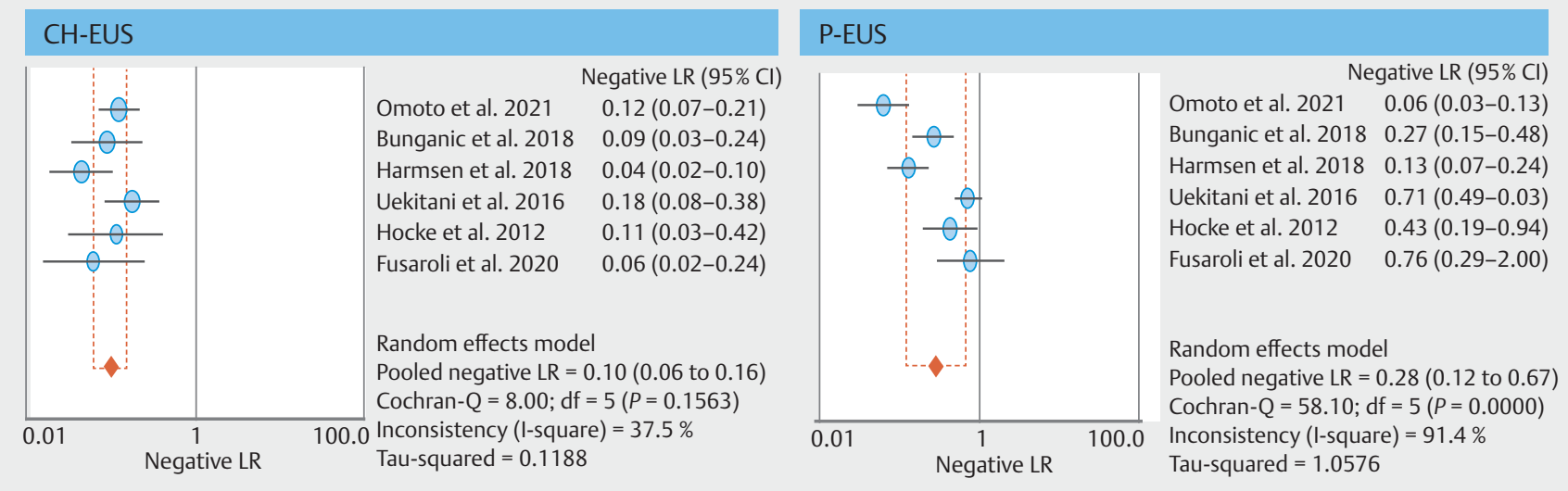

- Fig. 6 Forest plots of the pooled negative likelihood ratios on contrast-enhanced harmonic endoscopic ultrasonography (CH-EUS) and plain EUS (P-EUS). 

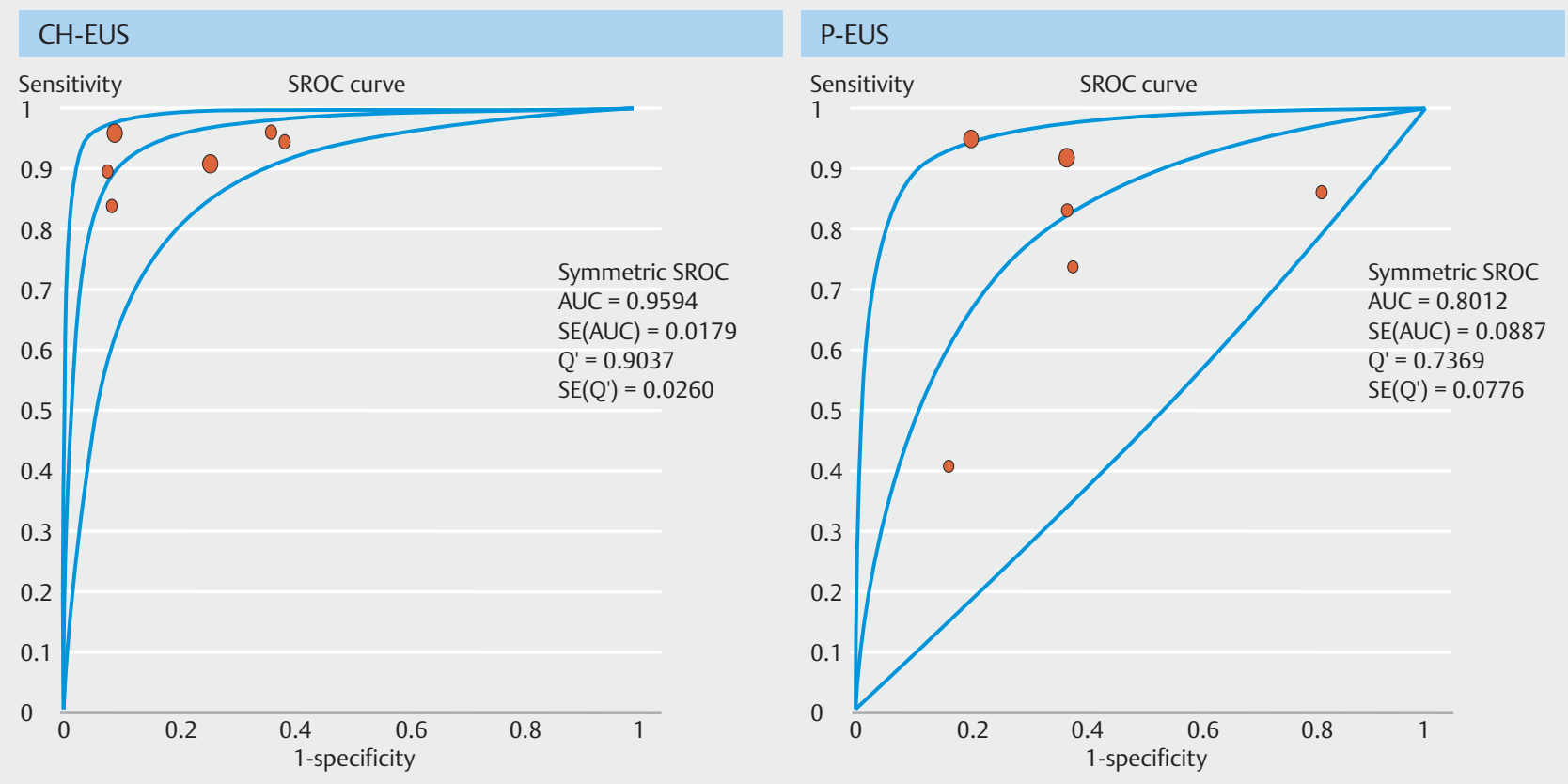

- Fig.7 Summary receiver operating characteristics (SROC) curves for accuracy of characterization for pancreatic tumors with contrast-enhanced harmonic endoscopic ultrasonography (CH-EUS) and plain EUS (P-EUS). AUC, area under the curve; SE, standard error; Q, the point at which sensitivity and specificity are equal.
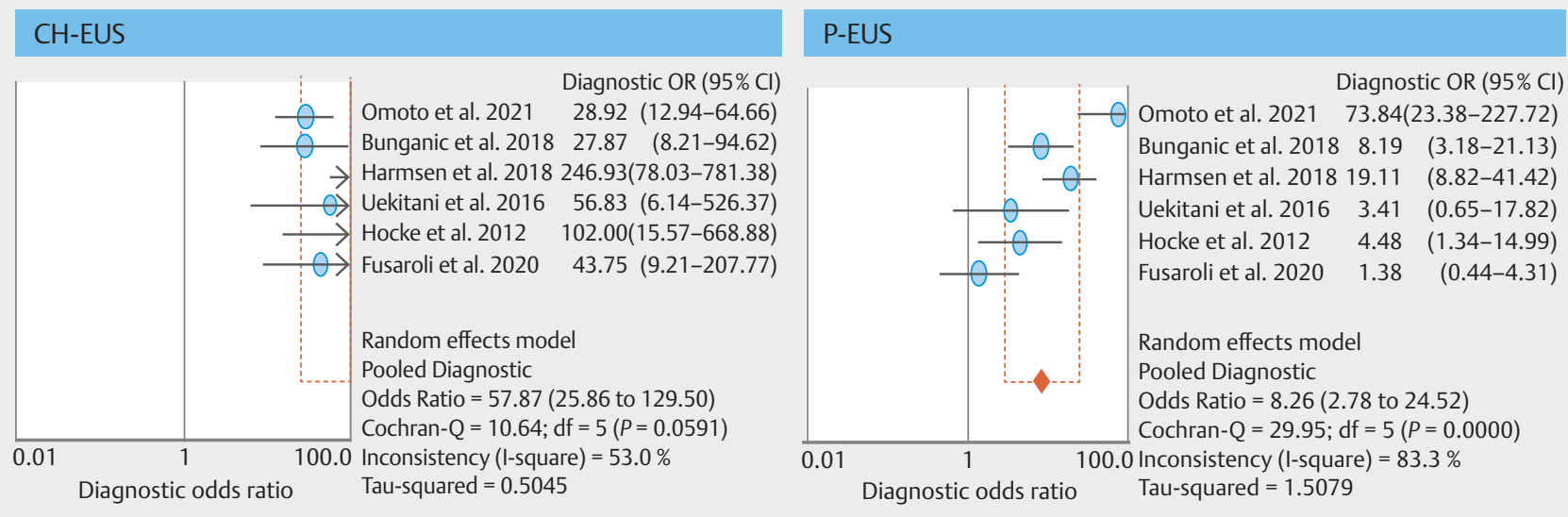

- Fig. 8 Forest plot of odds ratios for characterization for pancreatic tumors by contrast-enhanced harmonic endoscopic ultrasonography (CH-EUS) and plain EUS (P-EUS).

sensitivity, specificity, and SROC curve values of $91 \%$ to $94 \%$, $80 \%$ to $89 \%$, and $96 \%$ to $97 \%$, respectively, which are consistent with those reported in the present study [11-14]. However, because previous meta-analyses investigated only the diagnostic accuracy of $\mathrm{CH}$-EUS, it was not possible to determine whether $\mathrm{CH}$-EUS added something to P-EUS. $\mathrm{CH}$-EUS has some shortcomings compared with P-EUS. For instance, $\mathrm{CH}$-EUS involves additional costs, such as the cost of the ultrasound contrast agent and intravenous injection of it. Although adverse reactions to $\mathrm{CH}$-EUS contrast agents are rare in humans [23], injection of contrast agent also has risks of adverse events. Therefore, we considered it necessary to determine whether it was worth performing $\mathrm{CH}$-EUS as an additional test for characterization of pancreatic tumors. Articles in which both $\mathrm{CH}$-EUS and P-EUS were performed on the same patients were selected for this meta-analysis, which showed $\mathrm{CH}$-EUS to have pooled sensitivity, specificity, diagnostic odds ratio, and area under the SROC of $93 \%, 80 \%, 57.9$, and 0.96 , respectively, for the characterization of pancreatic tumors, whereas P-EUS showed pooled sensitivity, specificity, diagnostic odds ratio, and area under the SROC curve of $86 \%, 59 \%, 8.3$, and 0.80 , respectively. Moreover, the diagnostic odds ratio for accuracy of pancreatic cancer on $\mathrm{CH}$-EUS was 2.98 times higher than that on P-EUS, and $\mathrm{CH}$-EUS had statistically higher accuracy than P-EUS $(P=$ 


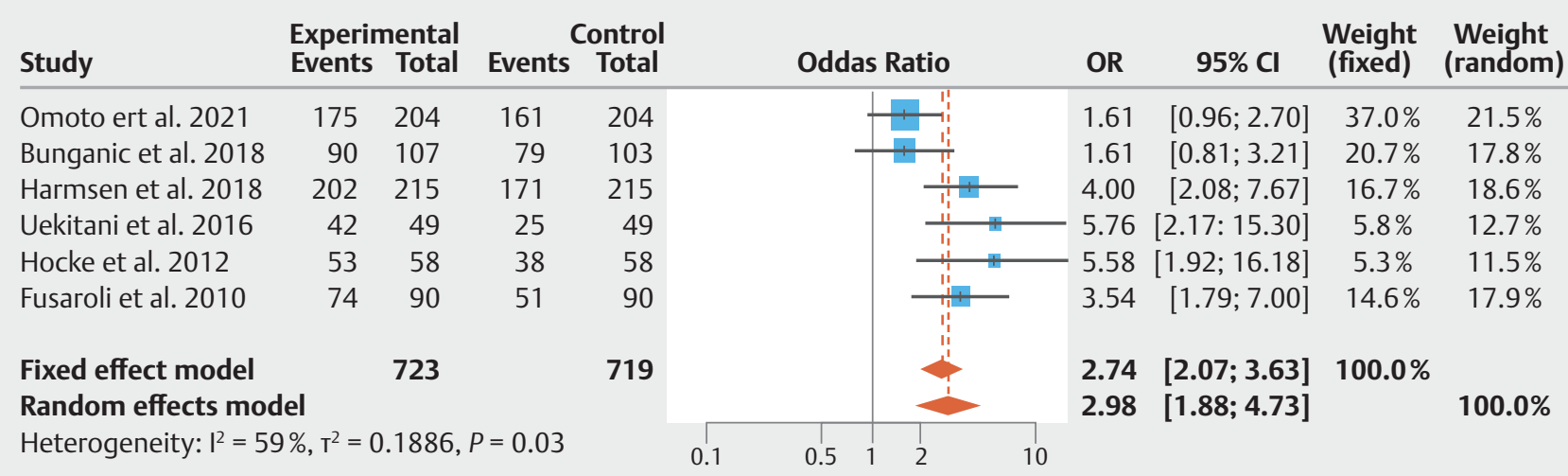

- Fig. 9 Comparison of the accuracy of contrast-enhanced harmonic endoscopic ultrasonography (CH-EUS) and plain EUS (P-EUS) for the characterization of pancreatic tumors.

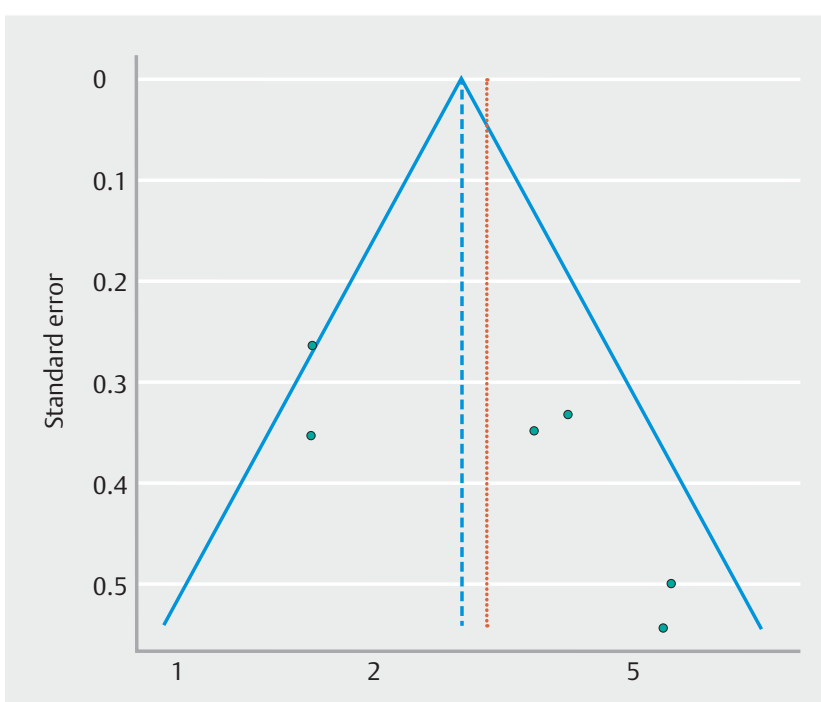

Fig. 10 Funnel plot to evaluate publication bias in the included studies.
0.03). The QUADAS-2 assessment showed that most studies had a low risk of bias, and assessment using funnel plots showed no publication bias. These results indicate that during EUS, CE-EUS should be used for the characterization of pancreatic tumors, in addition to P-EUS.

P-EUS offers advantages over other imaging modalities for detection and analysis of pancreatic cancer. Considering our findings indicating that $\mathrm{CH}$-EUS is superior to P-EUS, use of $\mathrm{CH}$-EUS may result in further improvement in diagnostic ability compared with contrast-enhanced MDCT. In fact, two articles reported that $\mathrm{CH}$-EUS was significantly superior to contrast-enhanced MDCT and/or MRI for characterization of small pancreatic tumors $(\leq 20 \mathrm{~mm})$ in clinical practice $[9,10]$. Another advantage is that CH-EUS is superior to contrast-enhanced MDCT and contrast-enhanced MRI in patients with contraindications, such as renal failure or iodine contrast allergy, because adverse reactions to $\mathrm{CH}$-EUS contrast agents are less frequent in humans. In addition, it allows real-time dynamic imaging and repeated examinations, and does not expose the patient to ionizing radiation. Therefore, these facts and our findings suggest that in cases in which P-EUS detects pancreatic tumors that are too small to be found with other imaging modalities, $\mathrm{CH}$ EUS should be used for characterization of pancreatic tumors.

- Table 3 Summary of meta-analysis results.

\begin{tabular}{|c|c|c|}
\hline & CH-EUS (95\% Cl) & P-EUS (95\% Cl) \\
\hline Pooled sensitivity & $0.93(0.90,0.95)$ & $0.86(0.82,0.89)$ \\
\hline Pooled specificity & $0.80(0.75,0.85)$ & $0.59(0.52,0.65)$ \\
\hline Pooled positive likelihood ratio & $4.70(2.70,8.21)$ & $2.16(1.20,3.87)$ \\
\hline Pooled negative likelihood ratio & $0.10(0.06,0.16)$ & $0.28(0.12,0.67)$ \\
\hline Pooled diagnostic odds ratio & $57.87(25.86,129.50)$ & $8.26(2.78,24.52)$ \\
\hline Area under the SROC curve & 0.959 & 0.801 \\
\hline
\end{tabular}


Currently, when a pancreatic tumor is detected on P-EUS, EUS-TA is performed to establish a definitive diagnosis. Although EUS-TA is an important tool for pathological diagnosis of pancreatic tumors, it is subject to some limitations. First, it is difficult to perform EUS-TA when blood vessels intervene across the puncture line. A multicenter study showed that complications associated with EUS-TA (1.7\% of all interventions) are related to bleeding $(49.1 \%)$, pancreatitis (26.5\%), peritonitis (3.4\%), perforation (2.1\%), pancreatic duct leakage (3.4\%), and needle tract seeding (3\%) with EUS-TA [24]. Another limitation is presence of false-negative findings on EUS-TA. EUS-TA for identification of pancreatic cancers of $11 \mathrm{~mm}$ to $20 \mathrm{~mm}$ showed sensitivity, specificity, and accuracy of $75.9 \%$ to $92 \%$, $93.8 \%$ to $100 \%$, and $78.9 \%$ to $95 \%$, respectively [25], whereas for pancreatic cancer $\leq 10 \mathrm{~mm}$, Siddiqui et al. reported sensitivity, specificity, and accuracy of only $40 \%, 80 \%$, and $47.4 \%$, respectively [26]. EUS-TA may give false-negative results in patients with small pancreatic tumors. In these cases, $\mathrm{CH}$-EUS can complement characterization of pancreatic tumors. Gincul et al. reported that $\mathrm{CH}$-EUS and EUS-TA had accuracy of $95 \%$ and $96 \%$, respectively; sensitivity of $94 \%$ and $95 \%$; and specificity of $93 \%$ and $100 \%$, with there being no significant differences between the two modalities in clinical practice [27]. Nevertheless, five false-negative cases on EUS-TA were correctly classified by $\mathrm{CH}$-EUS. Therefore, we propose the following strategy for using $\mathrm{CH}$-EUS. EUS for management of masses of the pancreas should include standard EUS, CH-EUS, and then EUS-TA. If the hypoechogenic mass is hypo-enhanced on $\mathrm{CH}$-EUS and the sample is not a cancer, then we quickly reschedule a second sampling session; conversely, if the hypo-echogenic mass is not hypo-enhanced on $\mathrm{CH}$-EUS and the sample shows no evidence of a tumor (only inflammatory tissue with fibrosis), we are confident in the hypothesis of a mass-forming pancreatitis. We then follow up the mass. Moreover, $\mathrm{CH}$-EUS can be an alternative diagnostic method for small tumors for which it is difficult or impossible to perform EUS-TA.

This study has some limitations. First, no randomized controlled trials were included, and the study designs were retrospective in $50 \%$ of the selected studies. Second, heterogeneity in the specificity and the positive likelihood ratio might have affected interpretation of the data and conclusions. However, a random effects model (DerSimonian-Laird method) was used when statistically significant heterogeneity was detected. Third, exclusion of conference abstracts, case reports, reviews, and unpublished data may have given rise to publication bias, such that our results may have overestimated the actual diagnostic performance.

\section{Conclusions}

In conclusion, the results of this study demonstrate that $\mathrm{CH}$ EUS provides significantly better diagnostic accuracy for pancreatic cancer than P-EUS in clinical practice.

\section{Competing interests}

Kitano and Napoléon have received honoraria from Olympus Corporation for presentations at conferences. Dr. Dietrich has received honoraria from Hitachi and Pentax Corporations for presentations at conferences.

References

[1] American Cancer Society. Cancer Facts \& Figures 2017. Atlanta: American Cancer Society; 2017

[2] The Editorial Board of the Cancer Statics in Japan, Foundation for Promotion of Cancer Research. Cancer Statics in Japan-2015. Tokyo, Japan: FPCR c/o National Cancer Center; 2016: 1-129

[3] Rawla P, Sunkara T, Gaduputi V. Epidemiology of pancreatic cancer: global trends, etiology and risk factors. World J Oncol 2019; 10: 1027

[4] Ahmad NA, Kochman ML, Lewis JD et al. Endosonography is superior to angiography in the preoperative assessment of vascular involvement among patients with pancreatic carcinoma. J Clin Gastroenterol 2001; 32: 54-58

[5] DeWitt ], Devereaux B, Chriswell M et al. Comparison of endoscopic ultrasonography and multidetector computed tomography for detecting and staging pancreatic cancer. Ann Intern Med 2004; 141: 753-763

[6] Krishna SG, Rao BB, Ugbarugba E et al. Diagnostic performance of endoscopic ultrasound for detection of pancreatic malignancy following an indeterminate multidetector $\mathrm{CT}$ scan: a systemic review and meta-analysis. Surg Endosc 2017; 31: 4558-4567

[7] Lee TY, Cheon YK, Shim CS et al. Clinical role of contrast-enhanced harmonic endoscopic ultrasound in differentiating solid tumors of pancreas: A single-center experience in Korea. Gut Liver 2013; 7: 559-604

[8] Dietrich CF, Sahai AV, D'Onofrio M et al. Differential diagnosis of small solid pancreatic tumors. Gastrointest Endosc 2016; 84: 933-940

[9] Kitano M, Kudo M, Yamao K et al. Characterization of small solid tumors in the pancreas: The value of contrast-enhanced harmonic endoscopic ultrasonography. Am J Gastroenterol 2012; 107: 303-310

[10] Yamashita Y, Tanioka K, Kawaji Y et al. Utility of contrast-enhanced harmonic endoscopic ultrasonography for early diagnosis of small pancreatic cancer. Diagnostics 2020; 10: 23

[11] Yamashita Y, Shimokawa T, Napoléon B et al. Value of contrast-enhanced harmonic endoscopic ultrasonography with enhancement pattern for diagnosis of pancreatic cancer: A meta-analysis. Dig Endosc 2019; 31: 125-133

[12] He XK, Ding Y, Sun LM. Contrast-enhanced endoscopic ultrasound for differential diagnosis of pancreatic cancer: an updated meta-analysis. Oncotarget 2017; 8: 66392-66401

[13] Gong TT, Hu DM, Zhu Q. Contrast-enhanced EUS for differential diagnosis of pancreatic mass tumors: a metaanalysis. Gastrointest Endosc 2012; 76: 301-309

[14] Mei S, Wang M, Sun L. Contrast-enhanced EUS for differential diagnosis of pancreatic masses: a meta-analysis. Gastroenterol Res Pract 2019; 6: 1670183

[15] Mantel N, Haenszel W. Statistical aspects of the analysis of data from retrospective studies of disease. J Natl Cancer Inst 1995; 22: 719-748

[16] DerSimonian R, Laird N. Meta-analysis in clinical trials. Control Clin Trials 1986; 7: 177-188

[17] Fusaroli P, Spada A, Mancino MG et al. Contrast harmonic echoendoscopic ultrasound improves accuracy in diagnosis of solid pancreatic masses. Clin Gastroenterol Hepatol 2010; 8: 629-634 
[18] Uekitani K, Kaino S, Harima $\mathrm{H}$ et al. Efficacy of contrast-enhanced harmonic endoscopic ultrasonography in the diagnosis of pancreatic ductal carcinoma. Saudi J Gastoroenterol 2016; 22: 198-202

[19] Bunganič B, Laclav M, Dvořáková T et al. Accuracy of EUS and CEH EUS for the diagnosis of pancreatic tumours. Scand J Gastroenterol 2018; 53: $1411-1417$

[20] Harmsen FR, Domagk D, Dietrich CF et al. Discriminating chronic pancreatitis from pancreatic cancer: Contrast-enhanced EUS and multidetector computed tomography in direct comparison. Endosc Ultrasound 2018; 7: 395-403

[21] Hocke M, Ignee A, Dietrich CF. Advanced endosonographic diagnostic tools for discrimination of focal chronic pancreatitis and pancreatic carcinoma - elastography, contrast enhanced high mechanical index (CEHMI) and low mechanical index (CELMI) endosonography in direct comparison. Z Gastroenterol 2012; 50: 199-203

[22] Omoto S, Kitano M, Fukasawa M et al. Tissue harmonic versus contrast-enhanced harmonic endoscopic ultrasonography for the diagnosis of pancreatic tumors: a prospective multicenter study. Dig Endosc 2021: doi:10.1111/den.13944
[23] Moriysu F, Itoh K. Efficacy of perflubutane microbubble-enhanced ultrasound in the characterization and detection of focal liver tumors: phase 3 multicenter clinical trial. AJR Am J Roentgenol 2009; 193: 8695

[24] Kanno A, Yasuda I, Irisawa A et al. Adverse events of endoscopic ultrasound-guided fine-needle aspiration for histologic diagnosis in Japanese tertiary centers: Multicenter retrospective study. Dig Endosc 2021; 7: 1146-1157

[25] Yamashita Y, Kitano M, Ashida R. Value of endoscopy for early diagnosis of pancreatic carcinoma. Dig Endosc 2020; 32: 27-36

[26] Siddiqui AA, Brown LJ, Hong SK et al. Relationship of pancreatic mass size and diagnostic yield of endoscopic ultrasound-guided fine needle aspiration. Dig Dis Sci 2011; 56: 3370-3375

[27] Gincul R, Palazzo M, Pujol B et al. Contrast-harmonic endoscopic ultrasound for the diagnosis of pancreatic adenocarcinoma: a prospective multicenter trial. Endoscopy 2014; 46: 373-379 